\title{
REVIEW
}

\section{Targeting Mitochondrial Function for the Treatment of Acute Spinal Cord Injury}

\author{
Melanie L. McEwen, ${ }^{1,2,3}$ Patrick G. Sullivan, ${ }^{1,3}$ Alexander G. Rabchevsky, ${ }^{1,4}$ \\ and Joe E. Springer ${ }^{1,2,3}$ \\ ${ }^{1}$ Spinal Cord and Brain Injury Research Center, ${ }^{2}$ Department of Physical Medicine and Rehabilitation, ${ }^{3}$ Department of Anatomy \\ and Neurobiology, ${ }^{4}$ Department of Physiology, University of Kentucky, Lexington, Kentucky 40536-0509
}

\begin{abstract}
Summary: Traumatic injury to the mammalian spinal cord is a highly dynamic process characterized by a complex pattern of pervasive and destructive biochemical and pathophysiological events that limit the potential for functional recovery. Currently, there are no effective therapies for the treatment of spinal cord injury (SCI) and this is due, in part, to the widespread impact of the secondary injury cascades, including edema, ischemia, excitotoxicity, inflammation, oxidative damage, and activation of necrotic and apoptotic cell death signaling events. In addition, many of the signaling pathways associated with these cascades intersect and initiate other secondary injury events. Therefore, it can be argued that therapeutic strategies targeting a specific biochemical cascade may not provide the best approach for promoting functional recovery. A "systems
\end{abstract}

approach" at the subcellular level may provide a better strategy for promoting cell survival and function and, as a consequence, improve functional outcomes following SCI. One such approach is to study the impact of SCI on the biology and function of mitochondria, which serve a major role in cellular bioenergetics, function, and survival. In this review, we will briefly describe the importance and unique properties of mitochondria in the spinal cord, and what is known about the response of mitochondria to SCI. We will also discuss a number of strategies with the potential to promote mitochondrial function following SCI. Key Words: Electron transport chain, mitochondria, permeability transition, reactive oxygen species, spinal cord injury, therapeutic strategies.

\section{MITOCHONDRIAL STRUCTURE AND ATP SYNTHESIS}

Mitochondria are double-membraned organelles that primarily function in oxidative phosphorylation. Specifically, mitochondria are the powerhouses of cells, taking products from the citric acid cycle (Krebs cycle), fatty acid oxidation, and amino acid oxidation, and producing most of the cell's supply of adenine triphosphate (ATP), which is the energy source used to power virtually all cellular functions. In fact, in the cells of evolutionarily "higher animals," $>95 \%$ of all ATP is produced by oxidative phosphorylation within mitochondria. Structurally, the mitochondrial outer membrane is a relatively simple phospholipid bilayer that contains porin-like

Electronic supplementary material The online version of this article (doi:10.1007/s13311-011-0031-7) contains supplementary material, which is available to authorized users.

Address correspondence and reprint requests to: Joe E. Springer, Ph. D., Spinal Cord and Brain Injury Research Center, University of Kentucky, Lexington, KY 40536-0509. E-mail: jspring@uky.edu. structures known as voltage-dependent anion channels (VDAC), protein structures that, when in the open state, allow molecules of approximately $5 \mathrm{kDa}$ or less in size to freely pass through the membrane, including ions, nutrients, adenine diphosphate (ADP), and ATP [1]. In contrast, the mitochondrial inner membrane is a highly complex structure that is freely permeable only to oxygen, carbon dioxide, and water. This membrane contains several intramembrane channels, which regulate the passage of all other molecules, and the electron transport chain (ETC), which functions to establish and maintain the electrochemical (proton) gradient across the inner membrane that is necessary for ATP synthesis [2].

The ETC is composed of four membrane-bound complexes (complexes I-IV) and two carrier molecules: coenzyme Q and cytochrome c (FIG. 1). The reduced form of nicotinamide adenine dinucleotide (NADH) is the primary reducing agent of the ETC, and complex I (NADH dehydrogenase) removes one pair of electrons at a time from the available NADH pool. As the electrons move to a lower energy state, complex I uses the free energy to pump 


\section{MITOCHONDRIAL OUTER MEMBRANE}

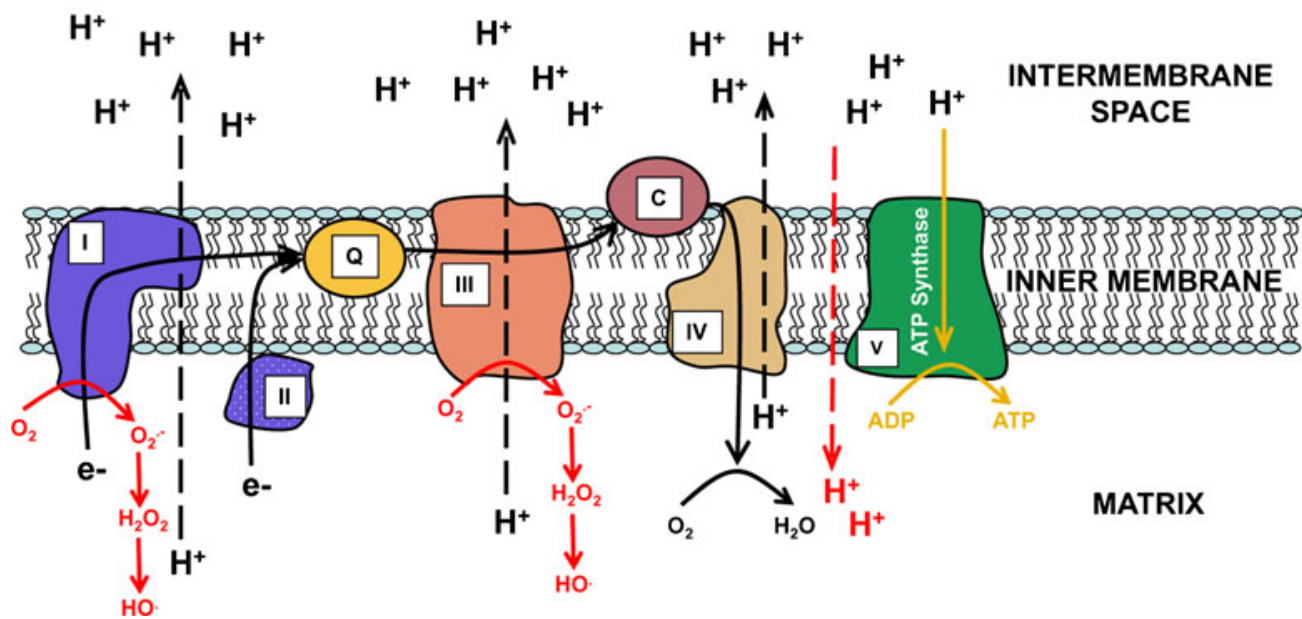

FIG. 1. Electron movement is shown through the mitochondrial electron transport chain (ETC) and the subsequent generation of adenosine triphosphate (ATP). Electrons (e-) from reduced substrates enter the ETC through complex I or II and are then passed through complexes III and IV where they have reduced oxygen $\left(\mathrm{O}_{2}\right)$ to produce water $\left(\mathrm{H}_{2} \mathrm{O}\right)$ (solid black lines). As the electrons are moved from a high-energy state to a low-energy state through the complexes, protons $\left(\mathrm{H}^{+}\right)$are pumped from the mitochondrial matrix into the intermembrane space (dashed black lines), generating a proton gradient across the mitochondrial inner membrane. Protons then re-enter the matrix through complex $\mathrm{V}$ and are used to combine adenosine diphosphate (ADP) with inorganic phosphate to produce ATP (solid yellow lines). Under homeostatic conditions, some electrons may leak from the respiratory chain and inappropriately combine with molecular oxygen (solid red lines), forming superoxide $\left(\mathrm{O}_{2}{ }^{-}\right)$, hydrogen peroxide $\left(\mathrm{H}_{2} \mathrm{O}_{2}\right)$, and hydroxyl radicals (HO) and contributing to steady-state levels of free radicals. Protons that leak back across the mitochondrial inner membrane and into the matrix (dashed red line) reduce the inner membrane potential, increasing the activity of the ETC and causing thermogenesis. $Q=$ coenzyme $Q ; C=c y t o c h r o m e ~ c$.

protons out of the matrix and into the intermembrane space at a proton-translocation stoichiometry of 4 protons for every 2 electrons, establishing a proton gradient across the mitochondrial inner membrane. In fact, electrons cannot pass through complex I without proton translocation. The electron pairs are then transferred to coenzyme Q, a lipidsoluble carrier, located within the mitochondrial inner membrane. Complex II (succinate dehydrogenase) of the ETC removes pairs of electrons from succinate; however, electrons from this "substrate couple" are at a lower energy state than those entering at complex I via NADH. Complex II-derived electron pairs are also delivered to the electron pool at coenzyme Q. After electrons are passed from coenzyme Q to complex III (cytochrome bc complex), they again move to a lower energy state, and the free energy is used to force additional protons out of the mitochondrial matrix and into the intermembrane space, enhancing the proton gradient across the mitochondrial inner membrane. The electron pairs are then transferred to cytochrome c, a water-soluble electron carrier located within the intermembrane space, and then to complex IV (cytochrome c oxidase). The free energy released as the electrons move to an even more lower energy state is used to pump even more protons into the intermembrane space, further strengthening the proton gradient across the inner membrane. The electron pairs are then used to reduce molecular oxygen in the matrix, producing water [2]. Single electrons that leak from the ETC may combine with molecular oxygen in the matrix to form superoxide, hydrogen peroxide, and hydroxyl radicals, contributing to the steady-state levels of free radicals. In mitochondria of the central nervous system, complex I appears to be the primary site from which electrons may prematurely leak from the ETC and inappropriately combine with oxygen, both under normal and pathological conditions [3].

The ATP synthase complex (often referred to as complex $\mathrm{V})$ is composed of 2 rotary motors $\left.\left(\mathrm{F}_{1}, \mathrm{~F}_{0}\right): 1\right)$ The hydrophilic domain $\left(\mathrm{F}_{1}\right)$ is suspended from the membrane into the mitochondrial matrix and contains the nucleotide binding and catalytic sites for ATP; this motor is driven by the energy released by the hydrolysis of ATP, and 2) the hydrophobic domain $\left(\mathrm{F}_{0}\right)$ is embedded within the mitochondrial inner membrane and consists of a proton channel, and it can be driven in the opposite direction by sufficient proton motive force. Thus, ATP synthesis (and ATP hydrolysis; see as follows) is coupled to proton movement through the membrane channel. Under homeostatic conditions, the proton gradient across the mitochondrial inner membrane forces protons from the intermembrane space, through the proton channel, and into the matrix; this proton movement coincides with a rotational change in the structure of the hydrophilic portion of the ATP synthase complex. When ADP and inorganic phosphate are available in the mitochondrial matrix, the series of conformational changes induced by proton movement $\left(F_{0}\right)$ forces the $F_{1}$ pump to run in its reverse direction so that the catalytic substrates can bind to their appropriate sites and ATP can be released [2, 4, 5]. Disruption in the activity of the respiratory complexes of the ETC results not only in an energy deficit 
(decreased ATP synthesis), but also increased production of reactive oxygen species (ROS) [3].

\section{MITOCHONDRIAL PERMEABILITY TRANSITION PORE}

Cell death following spinal cord injury (SCI) has been associated with multiple intracellular changes, including toxic elevations in the extracellular concentrations of excitatory and inhibitory amino acids, edema, inflammation, vascular dysfunction, and activation of proteases, such as calpains and caspases. Detailed discussion of these changes is beyond the scope of this review, and the reader is directed to several excellent reviews on the pathophysiological changes occurring in the spinal cord following injury [6-9]. However, of particular interest for this discussion are the ionic shifts that occur in the injured tissue that persist for hours and days following SCI [10-13]. In fact, within 15 to 60 minutes following SCI, the intracellular concentrations of sodium, chloride, and calcium $\left(\mathrm{Ca}^{2+}\right)$ increase, whereas the intracellular concentrations of potassium and magnesium decrease [14]. Mitochondria can function as $\mathrm{Ca}^{2+}$ "sinks," taking up the excess $\mathrm{Ca}^{2+}$ to maintain homeostatic levels of $\mathrm{Ca}^{2+}$ within the cytosol [15-18]. However, the principle trigger for opening the mitochondrial permeability transition pore (mPTP) in the mitochondrial inner membrane is mitochondrial matrix $\mathrm{Ca}^{2+}$ in the presence of phosphate.

As already mentioned, the mitochondrial inner membrane contains several intramembrane channels; opening of these channels is tightly regulated to prevent disruption of the mitochondrial inner membrane potential and ATP synthesis [19]. The mPTP is the most notorious of these channels, and opening of the MPTP has been linked to several different forms of cell death following SCI, including necrosis, apoptosis, autophagy, and necroptosis [19-23]. The molecular structure of the MPTP is still up for debate, but several lines of evidence previously suggested that VDAC in the mitochondrial outer membrane, adenine nucleotide translocator in the mitochondrial inner membrane, and cyclophilin D (Cyp-D) in the mitochondrial matrix were integral proteins that comprised the mPTP, at least in part [24-26]. However, recent studies with mitochondria from knockout mice have suggested that Cyp-D has more of a regulatory role in MPTP opening. In contrast, VDAC appears dispensable for pore formation, and at this time there is no compelling evidence that VDAC serves a regulatory role either [27].

The concentration of $\mathrm{Ca}^{2+}$ necessary to open the mPTP can depend on other factors. High $\mathrm{Ca}^{2+}$ levels are required when the inner membrane potential is strong, the matrix $\mathrm{pH}$ is low, or the ATP levels are high. Low $\mathrm{Ca}^{2+}$ levels may open the MPTP when the levels of ATP are extremely low, the inner membrane potential is reduced, or the matrix level of ROS is high [19]. Once the mPTP opens, the mitochondrial inner membrane can no longer serve as a barrier against proton flow, leading not only to the release of $\mathrm{Ca}^{2+}$ into the intermembrane space but also dissipation of the electrochemical gradient as the protons in the intermembrane space rush into the matrix, eventually causing a cessation of ATP synthesis [16]. In addition, mitochondria will actually begin to consume ATP. The mitochondrial inner membrane potential is approximately $150 \mathrm{mV}$ during ATP synthesis and catabolism, and ATP synthesis requires that the membrane potential remains at $80 \%$ to $90 \%$ of its maximum value [28]. When the inner membrane potential drops below that threshold, dissipation of the proton motive force (and cessation of $\mathrm{F}_{0}$ ) will allow the $F_{1}$ pump to reverse and run in its natural direction, and the energy released from ATP hydrolysis will drive the $\mathrm{F}_{0}$ pump in its reverse direction, forcing protons out of the matrix [5], exacerbating the energy deficit, and further disrupting mitochondrial homeostasis.

Pore opening allows any molecule with a molecular weight of $1.5 \mathrm{kDa}$ or less to enter the mitochondria and equilibrate across the inner membrane, a state known as mitochondrial permeability transition (mPT) [29-32]. The inrush of molecules and then water causes the mitochondrial matrix to swell as it equilibrates with the cytosol. The mitochondrial inner membrane has a much larger surface area than the outer membrane because of the cristae (folds) of the inner membrane. Matrix swelling expands the inner membrane outward until the more rigid outer membrane ruptures [33]. Rupture of the mitochondrial outer membrane releases all of the accumulated $\mathrm{Ca}^{2+}$ and $\mathrm{ROS}$, as well as numerous pro-apoptotic proteins (e.g., cytochrome c, apoptosis-inducing factor, SMAC/Diablo, and endonuclease-G) into the cytosol. Together, these substances can contribute to several forms of programmed cell death (i.e, any form of cell death mediated by intracellular machinery) including apoptosis, necroptosis, and autophagy [34-38]. However, activation and spread of these pathways is dependent on energy (ATP). If the function of "too many" mitochondria within a cell has been compromised, such that the cellular demand for energy outweighs the supply, the mode of cell death may be shifted away from programmed cell death and toward necrosis (oncosis), which proceeds passively in the absence of energy [39-42].

\section{MITOCHONDRIAL FUNCTION FOLLOWING SCI}

Assessment of mitochondrial bioenergetics is a valuable tool for studying not only the time course of mitochondrial failure following SCI, but also for shedding light on the underlying mechanisms responsible for the dysfunction. 
Oxygen is necessary to remove electrons from the ETC, because otherwise, all electron carriers would remain in a reduced state and electron transport (and ATP synthesis) would cease. Therefore, measurement of mitochondrial respiration (oxygen consumption) has been used to assess ETC function following SCI. Briefly, the oxygen consumption of isolated mitochondria is first measured at baseline (state I) and then following the addition of various compounds to the respiratory chamber to induce different respiratory states. The type of substrates and the order of their administration may vary slightly among laboratories, but the following sequence has worked well in our hands: 1) pyruvate plus malate, which are complex I substrates (state II); 2) ADP (state III); 3) oligomycin, an ATP synthase inhibitor (state IV); 4) carbonyl cyanide 4-(trifluoromethoxy) phenylhydrozone (FCCP), a mitochondrial uncoupler (state $\mathrm{V}$, complex I-driven respiration); and (5) rotenone, a complex I inhibitor, followed by succinate, a complex II substrate (state V, complex IIdriven respiration) [43-47]. Mitochondrial uncoupling, produced by FCCP, refers to a condition in which electron transport is functionally disconnected from the production of ATP $[48,49]$.

The respiratory control ratio (RCR), the ratio of the rate of oxygen consumption during state III to the rate of oxygen consumption during state IV, is one of the most sensitive measures of mitochondrial function, indicating the mitochondrial capacity to perform oxidative phosphorylation. Specifically, state III respiration reflects the maximal rate of coupled respiration, whereas state IV respiration reflects the rate of proton leakage from the mitochondrial intermembrane space into the matrix. Reports indicate that RCR values are comparable to control levels at 6 hours [47], but decreased at 12 hours [47] and 24 hours [43-47] following SCI, primarily due to a decrease in the rate of oxygen consumption following the addition of ADP (state III). Further investigation revealed that both complex I-driven [4347] and complex II-driven [43, 44] respiration was impaired postinjury and that mitochondrial oxidative stress was elevated [43, 44, 46, 47].

Together, these findings indicate that mitochondrial dysfunction following SCI is due to impaired functioning of both complexes I and II or a common downstream component of the ETC, increased ROS production, and decreased ATP synthesis. These findings are somewhat consistent with a recent study examining the effect of exogenously generated peroxynitrite on isolated spinal cord mitochondria [50]. In that study it was shown that NADH-linked respiration was the primary target of exogenous peroxynitrite generated by the peroxynitrite donor SIN-1. However, these authors did not report any effect of SIN-1 application on complex II, in contrast to what was seen in the SCI studies. This may be due to the possibility that peroxynitrite only targets certain compo- nents of the ETC, such as complex I. A second explanation is that the source (extra-mitochondrial vs intra-mitochondrial) and/or ratio of the free radicals may be vastly different between the different studies. Finally, there may be technical differences (in situ vs ex vivo) in the way the free radicals were generated or measured that could account for the reported differences. Regardless, it is clear from these studies that complex I of spinal cord mitochondria is highly sensitive to the presence of free radicals.

The remainder of this review will focus on the existing, as well as potential, treatment strategies for preserving mitochondrial function, with the goal of promoting cell survival and functional recovery following SCI. We will focus on 3 approaches to improve mitochondrial function: 1) use of alternate energy substrates, 2) administration of antioxidants, and 3) inhibition of mPTP formation. These strategies were chosen based on existing data in the literature, technical or pharmacological feasibility, and relative specificity to mitochondria.

\section{ALTERNATE ENERGY SUBSTRATES TO PROMOTE MITOCHONDRIAL FUNCTION}

As previously described, it is well-documented that mitochondrial function is significantly compromised within hours following SCI. NADH dehydrogenase (ETC complex I), cytochrome c oxidase (ETC complex IV), and pyruvate dehydrogenase (PDH) are key mitochondrial enzymes available to the mitochondrial matrix that are important in mitochondrial respiration, but subject to inactivation or decreased activity due to oxidative damage after SCI and other models of CNS injury. The significant loss of PDH activity following SCI limits the ability of pyruvate to generate acetyl coenzyme A ( $\mathrm{CoA})$, thus reducing the amount of acetyl-CoA available for the citric acid cycle (FIG. 2). Acetyl-CoA is critical for the production of NADH and $\mathrm{FADH}_{2}$ (reduced form of flavin adenine dinucleotide), which serve as electron donors for ATP synthesis when oxidized in the mitochondrial respiratory ETC. Given the loss of PDH after acute SCI, one approach is to introduce a substrate that can serve as an alternative source for energy production. Such an approach requires the identification of a substrate that is amenable to the unique molecular requirements of mitochondria, is readily available to the mitochondrial compartment of the cell, and, ideally, exhibits favorable pharmacokinetics with minimal side effects.

Acetyl-L-carnitine (ALC) is a natural component of the mitochondrial inner membrane that is synthesized from L-carnitine by ALC transferase in several organs, including the brain, liver, and kidneys. Several studies have documented that ALC can function as an alternative 
substrate for mitochondrial respiration and has been used to improve mitochondrial function and limit oxidative stress [51-56]. ALC promotes efficient energy production by facilitating the formation of acetyl-CoA in the absence of optimal PDH activity; this is accomplished, in part, when ALC donates acetyl groups to CoA directly such that the newly formed acetyl-CoA moiety can enter the citric acid cycle, effectively bypassing PDH (FIG. 2). A second attractive property of ALC is that it promotes the production of the antioxidant glutathione by donating a carbon from the acetyl moiety. This two-pronged approach is highly advantageous as a possible treatment strategy for SCI because ALC provides an alternative substrate source for the production of the much needed ATP, while simultaneously reducing the free radical load on the mitochondria. Two additional properties of ALC are that it readily crosses the blood-brain barrier and undergoes limited metabolism, making it a highly attractive candidate as an alternative energy substrate.

A recent report revealed that ALC could function as an alternative substrate for energy metabolism following acute SCI [45]. The results of this study showed that mitochondria isolated from the intact spinal cord were

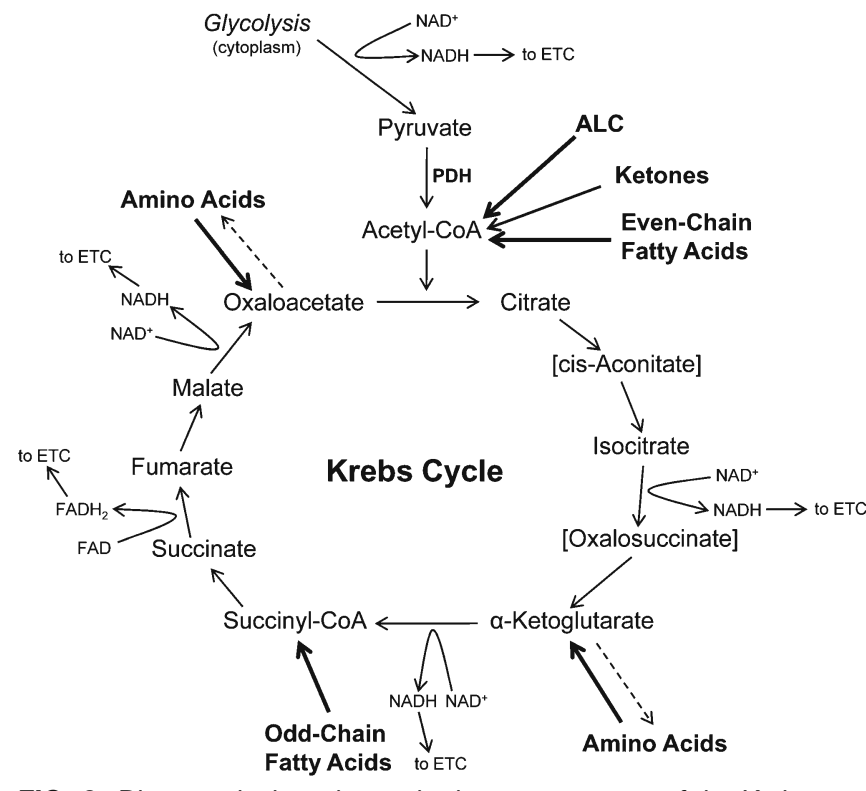

FIG. 2. Diagram depicts shows the key components of the Krebs (citric acid) cycle. The Krebs cycle is a series of enzyme-catalyzed chemical reactions within the matrix of mitochondria that, in conjunction with the electron transport chain (ETC), generates the energy that can be utilized by the cell. Acetyl-L-carnitine (ALC) can serve as an alternate energy source by promoting the formation of acetyl coenzyme $A(C \circ A)$ in the absence of optimal pyruvate dehydrogenase $(\mathrm{PDH})$ activity, leading to the reduction of nicotinamide adenine dinucleotide $\left(\mathrm{NAD}^{+}\right)$and flavin adenine dinucleotide (FAD). The reduced forms of $\mathrm{FAD}\left(\mathrm{FADH} \mathrm{H}_{2}\right)$ and $\mathrm{NAD}^{+}(\mathrm{NADH})$ serve as electron carriers to promote function of the ETC and the production of ATP. Other substances, such as amino acids (e.g., glutamate), ketones, and fatty acids may also potentially serve as alternative energy sources, entering the Krebs cycle at different points than ALC, but their efficacies have not been evaluated in spinal cord injury. capable of using ALC as an alternative and efficient substrate for mitochondrial respiration. More importantly, ALC restored respiratory activity to near normal levels in mitochondria isolated at $24 \mathrm{~h}$ following SCI. Given that PDH activity is compromised at this time point, thus reducing the ability of the respiratory chain to use pyruvate, the demonstration that ALC is an efficient substitute substrate is promising. Finally, ALC treatment postinjury significantly improved mitochondrial respiration and promoted gray matter tissue sparing. The use of ALC as a treatment for acute SCI is early in its development. However, the results of this initial study are promising, and based on the safety profile of ALC [57] there is a high potential for translation to the clinic. Although other substrates, such as ketones, amino acids (e.g., glutamate), and fatty acids, may also be metabolized into intermediates of the citric acid cycle (see FIG. 2), the efficacy of these substances as alternative energy substrates has not been examined in SCI.

\section{DEVELOPMENT OF ANTIOXIDANT STRATEGIES DESIGNED TO TARGET MITOCHONDRIA}

Mitochondria continuously produce free radicals (e.g., superoxide) that are neutralized by endogenous antioxidants, including superoxide dismutase and glutathione. In fact, estimates suggest that as much as 2 to $5 \%$ of the total oxygen that interacts with the ETC is incompletely reduced to form ROS [58, 59]. However, under pathological conditions, such as those that occur following SCI, free radical production exceeds the neutralizing capacity of these naturally occurring antioxidants $[8,58]$. As a consequence, these oxyradicals accumulate and contribute to the formation of lipid peroxidation, protein oxidation, and peroxynitrite byproducts that interact with and inactivate critical mitochondrial enzyme complexes. One approach to combat the increase in free radical accumulation is to develop modified antioxidants that target the mitochondrial matrix, where these oxyradicals are produced $[18,58-60]$. A separate review dedicated to antioxidant treatment in acute SCI is included in this issue and the reader is referred the accompanying review by Hall et al. for details. Here, we will briefly summarize some of the major findings in the context of identifying appropriate antioxidant candidates for mitochondrial targeting.

One of the first antioxidants to be studied for the treatment of acute SCI was alpha-tocopherol, which is a fat-soluble lipid peroxyl scavenger with vitamin E-like activity. Pretreatment with alpha-tocopherol, along with continued postinjury treatment, significantly improved functional recovery in cats that sustained a compression injury to spinal cord level L2 [61]. Similar findings were 
reported in a more recent study using postinjury alphatocopherol treatment in a rat model of spinal cord compression [62]. Based on its demonstrated lipid peroxyl scavenging activity, the improvement in functional recovery was associated with a decrease in the lipid peroxidation product malondialdehyde [63]. Although effective in some experimental models of SCI, the use of alpha-tocopherol alone has several disadvantages, including a lack of mitochondrial specificity and potential harmful side effects at high doses [64, 65].

A second antioxidant approach for the treatment of SCI is the use of spin trap molecules. Broadly defined, spin trapping involves the addition of a free radical to a nitrone (i.e., the spin trap), resulting in the formation of a nitroxide-based persistent radical referred to as a spin adduct. The most widely recognized spin traps are alphaphenyl N-tertiary-butyl nitrone (PBN) and 5,5-dimethylpyrroline N-oxide. Although spin traps are commonly used for identifying free radical species via electron spin resonance, they also serve a biological role as stabilizers of ROS generated in cells undergoing oxidative stress $[66,67]$. For example, intravenous PBN treatment has been shown to improve energy metabolism in the injured spinal cord, possibly through the reduction of free radical-mediated mitochondrial dysfunction [68]. However, in a follow-up study by this same group, PBN pretreatment had no effect on functional recovery or axonal integrity [69]. In this latter study, PBN was administered in a different fashion (intraperitoneal) compared to the intravenous route used in the energy metabolism study. Therefore, it is not clear whether the treatment paradigm used in the functional study allowed for differences in the pharmacokinetic profile of PBN using these two routes of administration.

Tempol (4-hydroxy-2,2,6,6-tetramethylpiperidine- $N$ oxyl) is a nitroxide spin trap molecule that has been examined in a number of in vitro and in vivo models of oxidative damage. Several properties make it an ideal antioxidant for mitochondrial targeting, including its high cell permeability and broad-acting neutralizing activity against several ROS, including superoxide, hydroxyl radicals, and peroxynitrite-derived free radicals. In comparable models of SCI, tempol has been shown to improve functional recovery and tissue sparing [70], reduce measures of oxidative damage [46] and cytoskeletal disruption [71], and promote mitochondrial function [71]; however, see Patel et al. [46].

Neu2000 (2-hydroxy-5-[2,3,5,6-tetrafluoro-4-trifluoromethyl-benzylamino]-benzoic acid) is a recently characterized compound synthesized from acetylsalicylic acid and sulfasalazine and, based on its electron spin resonance spectra, is a novel spin trap molecule. Acetylsalicylic acid has been shown to significantly reduce NMDA receptor-mediated excitotoxicity through a mechanism involving inhibition of nuclear factor- $\mathrm{kB}$ and c-Jun N-terminal kinase [72, 73]. Neu2000 acts as an NR2B-receptor-specific, low-affinity gating modifier in an uncompetitive manner that enhances NMDA desensitization and stabilizes the closed state of NMDA receptors [74]. Sulfasalazine, another anti-inflammatory drug, also functions as a scavenger of free radicals $[75$, 76]. Neu2000 was generated by combining these two compounds, resulting in dual neuroprotective actions by functioning as an uncompetitive NMDA receptor antagonist and a free radical scavenger. In a recently published study, Neu2000 reduced ROS production in isolated mitochondria obtained at $24 \mathrm{~h}$ following spinal cord contusion [77]. Specifically, isolated mitochondria produced significantly elevated levels of ROS when the mitochondrial membrane potential was maximized by adding oligomycin to the respiratory chamber, a pathophysiological state that mimics the environmental stressors present in the injured spinal cord [47, 49, 78]. This observation provides indirect in vivo evidence that Neu2000 functions as a potent free radical scavenger, which is consistent with its property as a spin trap.

Although these antioxidant molecules exhibit neuroprotective properties, their distribution in the body following systemic administration limits their efficacy on the mitochondria. This finding has led to the generation of a new class of chimeric compounds that share 2 important properties: 1) free radical scavenging and 2) a high selectivity for the mitochondrial matrix in which the vast majority of free radicals are generated. These bioactive molecules are generated when potent antioxidants are covalently coupled to mitochondrial targeting compounds. One such compound is triphenylphosphonium cation (TPP), which has been used for measuring the mitochondrial inner membrane potential. Estimates suggest that linking antioxidants to TPP increases their accumulation in the mitochondrial matrix by several hundred-fold [79-81]. Examples of such mitochondria-targeted antioxidants include MitoVitE (TPP coupled to alpha-tocopherol), Mito-Q (TPP coupled to ubiquinone), Mito-PBN (TPP coupled to PBN), and Mito-CP (TPP coupled to carboxy-proxyl nitroxide). Coupling TPP to tempol or Neu2000 has not been investigated, even though, given the high free radical scavenging activity of these compounds, such an approach is potentially very promising. Other mitochondrial targeting antioxidants include aromatic cationic peptides, called SS tetrapeptides, which contain the phenolic antioxidant 2',6'-dimethyltyrosine linked to alternating aromatic and basic amino acid residues. At least four SS tetrapeptides (SS-02, SS-19, SS-20, and SS-31) have been generated that exhibit potent and mitochondria-specific antioxidant properties [64]. Similar to the TPP-linked antioxidants, these SS tetrapeptides accumulate in mitochondria in amounts 
that are several-fold higher than the extracellular concentrations.

At the present time, the use of mitochondria-targeted antioxidants has not been described in models of SCI. However, given the high specificity of these molecules for targeting the mitochondrial matrix and their potent antioxidant properties, it is clear that future studies will reveal their therapeutic potential. In support of this notion, mitochondria-targeted antioxidants (i.e., 5,5dimethyl-pyrroline N-oxide, Mito-Q and Mito-CP) have been shown to reduce oxidative damage and improve mitochondrial function in the superoxide dismutase-1 (G93A) transgenic mouse model of amyotrophic lateral sclerosis [82].

\section{TARGETING THE MPTP IN ACUTE SCI}

As previously described, some of the events thought to significantly contribute to the pathophysiological changes following acute SCI include disruption of the mitochondrial inner membrane potential and formation of the mPTP, leading to $\mathrm{MPT}$ and cell death via necrosis [83-86], as well as programmed cell death mechanisms [87-93]. Therefore, promoting mitochondrial function by limiting $\mathrm{mPTP}$ formation and subsequent $\mathrm{MPT}$ may have significant therapeutic benefits for the treatment of acute SCI.

Numerous experimental studies and clinical observations have demonstrated that the immunosuppressant cyclosporin A (CsA), which inhibits $\mathrm{mPT}$ and promotes mitochondrial function, may be of therapeutic benefit in the treatment of acute traumatic brain injury (TBI), SCI, cerebral ischemia, and reperfusion injury following acute myocardial infarction [94-103]. Phase I clinical trials for the acute treatment of severe TBI have been performed, and follow-up trials have been proposed [104]. In addition, a recently completed clinical study reported that CsA administration resulted in a smaller infarct size in patients undergoing percutaneous coronary intervention [99]. However, a role for CsA as a neuroprotective agent in experimental models of acute SCI is inconclusive due to conflicting reports regarding its efficacy [105-110].

The reasons why CsA has not been proven to be effective in promoting neuroprotection in models of SCI compared to TBI are not clear. However, in existing SCI studies, a limited number of CsA dosing regimens have been tested compared to studies of TBI [111]. In addition, it is possible that differences in the effectiveness of CsA treatment for TBI and SCI may be due to inherent differences in the biological properties of the brain vs spinal cord mitochondria [78]. For example, significantly lower concentrations of $\mathrm{Ca}^{2+}$ induce $\mathrm{mPT}$ in spinal cord mitochondria compared to brain (cortical) mitochondria. This same study found that Cyp-D levels are significantly higher in spinal cord mitochondria relative to cortical mitochondria [78]. Based on these observations, it could be argued that higher doses of CsA may be required to obtain similar efficacy in models of SCI compared to TBI. In summary, it is difficult to draw conclusions on the potential efficacy of CsA for SCI from the results obtained for TBI. In addition, the welldocumented caveat that CsA is highly toxic and has potent immunosuppressive properties makes it a less than ideal therapeutic candidate for the treatment of acute SCI. Therefore, compounds that mimic the actions of CsA on $\mathrm{mPT}$, but exhibit a much lower cytotoxicity profile and significantly reduced immunosuppressive properties, would be more attractive as therapeutic candidates.

NIM811 ( $N$-methyl-isoleucine-cyclosporin) is a cyclosporin analog that also binds to Cyp-D and blocks mPT at nanomolar concentrations in brain mitochondria [112114]. NIM811 was originally developed to be a less toxic alternative to CsA, but the modifications made to the cyclosporin structure eliminated the immunosuppressive properties mediated by calcineurin inhibition. This latter property is a serendipitous advantage because it eliminates the immunosuppressive confound associated with CsA. At the present time, NIM811 and two other cyclophilin inhibitors are in clinical trials as co-treatments for hepatitis $\mathrm{C}$ virus (HCV). These compounds are thought to partially inhibit HCV replication by binding to cyclophilin A and possibly other cyclophilins, of which there are at least 16 expressed in mammalian cells [115-117]. Additional studies suggest that co-treatment with cyclophilin inhibitors and protease or polymerase inhibitors leads to a reduction in $\mathrm{HCV}$ replication and limits development of resistance to monotherapy alone [117-120].

A second advantageous property of NIM811 is that it exhibits a significantly lower cytotoxic profile relative to CsA. Both NIM811 and CsA prevent depolarization of the mitochondrial inner membrane and inhibit $\mathrm{mPT}$ induced by $\mathrm{Ca}^{2+}$ overload in mitochondria isolated from rat hepatocytes. However, NIM811 protects against tumor necrosis factor- $\alpha$-induced apoptosis, spanning a much wider concentration range compared to CsA [113]. The cytoprotective properties of NIM811 against apoptotic stimuli have also been reported in other cell types [113, $114,121,122]$. Therefore, the unique properties of NIM811 allows for testing of a broader dose range relative to CsA.

As with CsA, NIM811 does not easily cross the intact blood-brain barrier and presumably a similar pattern would be observed for the blood-spinal cord barrier. However, the blood-spinal cord barrier is severely disrupted at early times following injury [123, 124], and therapeutic concentrations of NIM811 are reached within 15 minutes and maintained in the spinal cord for up to $24 \mathrm{~h}$ postinjury following a single $40-\mathrm{mg} / \mathrm{kg}$ dose. Given that the blood-spinal cord barrier remains "leaky" for up to $72 \mathrm{~h}$ following injury, repeated dosing of NIM811 is feasible. 
Reliable and reproducible measures of mitochondrial function and respiration can be obtained in mitochondria isolated from the intact and injured spinal cord [43, 44, 46, 47]. In an initial study, the effects of NIM 811 treatment on mitochondrial bioenergetics and ROS production following acute SCI were examined [44]. In synaptic mitochondria isolated from spinal cord-injured rats, NIM811 treatment significantly improved mitochondrial RCRs and the maximal electron transport capacity of complexes I and II. In addition, NIM811 increased the ATP-producing capacity of mitochondria. Consistent with the improvements in mitochondrial function, mitochondria isolated from the neurons of spinal cord-injured animals treated with NIM811 exhibited a decrease in free radicals produced under conditions that maximize free radical output.

Based on these previous findings, it could be postulated that improvement in mitochondrial function would translate to a reduction in markers of apoptotic cell death at acute times following SCI. Such findings would be consistent with observations that formation of the MPTP and subsequent $\mathrm{mPT}$ are linked to the mitochondrial release of pro-apoptotic molecules. Therefore, additional "proof of concept" studies were conducted to examine the effects of NIM811 treatment on indices of apoptosis and tissue sparing at acute time points following SCI. In a follow-up study, NIM811 treatment reduced cytosolic levels of cytochrome $\mathrm{c}$ and fragmented DNA during the first $24 \mathrm{~h}$ following SCI and also enhanced the volume of spared gray and white matter at 7 days postinjury [125].

The neuroprotective effects of NIM811 have been documented in several other experimental models of cell death. For example, NIM811 treatment significantly reduced infarct volume, free radical production, and cytochrome c release in a model of transient forebrain cerebral ischemia $[126,127]$. In a recent study using an animal model of TBI, NIM811 treatment increased cortical tissue sparing, improved mitochondrial function, and reduced oxidative damage in adult rats [128]. In a follow-up study, the cytoprotective effects of NIM811 were similar to $\mathrm{CsA}$, providing strong evidence that the actions of both compounds function to maintain mitochondrial integrity [129]. Interestingly, in this same study, delaying NIM811 administration by $12 \mathrm{~h}$ following injury was effective in preventing cytoskeletal degradation. Finally, NIM811 significantly reduced reperfusion injury following experimental acute myocardial infarction [94, 96, 99]. Collectively, the findings of these studies suggest that an approach that targets the mPTP can be generalized to several models of cell death. More importantly, it can be argued that the actions of NIM811 are due primarily to its actions on mPTP formation, whereas calcineurin inhibition (attributed to the mechanisms of action of CsA) does not appear to play a major role.

\section{CONCLUSIONS}

The premise for this review is based on the understanding that mitochondrial function, which is critical for maintaining cellular bioenergetics and limiting ongoing cell death and dysfunction, is severely compromised following SCI. Therapies that have a high specificity and high selectivity for targeting mitochondrial function should prove beneficial in the treatment of acute SCI (see FIG. 3). It is appreciated that SCI is highly complex,

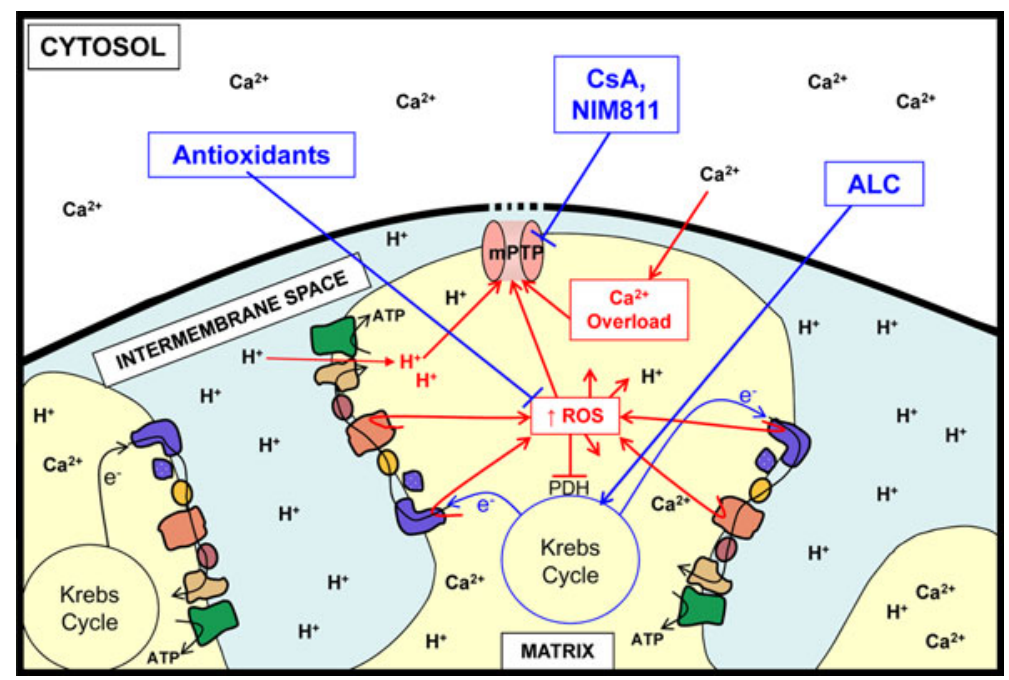

FIG. 3. This figure illustrates the presumed mitochondrial sites of action of the treatment strategies discussed throughout this review. Acetyl-L-carnitine (ALC) promotes energy (ATP) production by serving as an alternative energy source to the Krebs cycle. Antioxidants help to neutralize the deleterious effects of reactive oxygen species (ROS). Cyclosporin A (CsA) and $N$-methyl-isoleucine-cyclosporin (NIM811) promote mitochondrial function by blocking formation of the mitochondrial permeability transition pore $(\mathrm{mPTP})$. ATP=adenosine triphosphate; $\mathrm{Ca}^{2+}=$ calcium; $\mathrm{e}^{-}=$electrons; $\mathrm{H}^{+}=$protons; $\mathrm{PDH}=$ pyruvate dehydrogenase. 
encompasses several independent and interrelated pathophysiological events, and that no single approach is going to maximize recovery of function. However, most treatment strategies to date have focused on the inhibition or promotion of particular steps in one of many molecular signaling cascades, with little to no attention toward approaches that target entire organelles. In addition, a single treatment approach may not provide optimal mitochondrial protection or recovery following SCI, and it may be necessary to use a combination approach, such as NIM811 and ALC. Using such an approach would require a more detailed understanding of the time course of mitochondrial dysfunction as it pertains to the targeted therapy (e.g., mPT vs substrate replacement). Finally, the strategies discussed in this review are limited, as they are unable to target unique mitochondrial subpopulations. In certain circumstances, it may be important to identify approaches that are highly selective for synaptic (i.e., neuronal) vs nonsynaptic (e.g., glial, and so forth) mitochondrial subpopulations. Regardless, we have described several mitochondriatargeted strategies with clear clinical potential for the treatment of acute SCI. Some of these treatment strategies are in different stages of development, whereas others were presented to simply provide proof of principle to theoretical approaches that may lead to more highly refined treatments.

Acknowledgments: Preparation of the manuscript was funded by an endowment from Cardinal Hill Rehabilitation Hospital (J.E.S.) and a grant from the Craig H. Neilsen Foundation (M.L.M.). Full conflict of interest disclosure is available in the electronic supplementary material for this article.

\section{REFERENCES}

1. Lemasters JJ, Holmuhamedov E. Voltage-dependent anion channel (VDAC) as mitochondrial governator-thinking outside the box. Biochim Biophys Acta 2006;1762:181-190.

2. Saraste M. Oxidative phosphorylation at the fin de siecle. Science 1999;283:1488-1493.

3. Turrens JF. Mitochondrial formation of reactive oxygen species. J Physiol 2003;552:335-344.

4. Ackerman SH, Tzagoloff A. Function, structure, and biogenesis of mitochondrial ATP synthase. Prog Nucleic Acid Res Mol Biol 2005;80:95-133.

5. Oster G, Wang H. Reverse engineering a protein: the mechanochemistry of ATP synthase. Biochim Biophys Acta 2000;1458:482-510.

6. Ahn YH, Lee G, Kang SK. Molecular insights of the injured lesions of rat spinal cords: Inflammation, apoptosis, and cell survival. Biochem Biophys Res Commun 2006;348:560-570.

7. Genovese T, Cuzzocrea S. Role of free radicals and poly(ADPribose)polymerase-1 in the development of spinal cord injury: new potential therapeutic targets. Curr Med Chem 2008; 15:477-487.

8. Hall ED, Springer JE. Neuroprotection and acute spinal cord injury: a reappraisal. NeuroRx 2004;1:80-100.

9. Rowland JW, Hawryluk GW, Kwon B, Fehlings MG. Current status of acute spinal cord injury pathophysiology and emerging therapies: promise on the horizon. Neurosurg Focus 2008;25:E2.
10. Young W, Koreh I. Potssium and calcium changes in injured spinal cords. Brain Research 1986;365:42-53.

11. Demediuk P, Lemke M, Faden A. Spinal cord edema and changes in tissue content of $\mathrm{Na}+, \mathrm{K}+$, and $\mathrm{Mg} 2+$ after impact trauma in rats. Adv Neurol 1990;52:225-232.

12. Kwo S, Young W, Decrescito V. Spinal cord sodium, potassium, calcium, and water concentration changes in rats after graded contusion injury. J Neurotrauma 1989;6:13-24.

13. Sharma H, Winkler T, Stalberg E, Olsson Y, Dey P. Evaluation of traumatic spinal cord edema using evoked potentials recorded from the spinal epidural space. An experimental study in the rat. J Neurol Sci 1991;102:150-162.

14. LoPachin R, Gaughan C, Lehning E, Kaneko Y, Kelly T, Blight A. Experimental spinal cord injury: spatiotemporal characterization of elemental concentrations and water contents in axons and neuroglia. J Neurophysiol 1999;82:2143-2153.

15. Chinopoulos C, Adam-Vizi V. Mitochondrial Ca2+ sequestration and precipitation revisited. Febs J 2010;277:3637-3651.

16. Crompton $\mathrm{M}$. The mitochondrial permeability transition pore and its role in cell death. Biochem J 1999;341:233-249.

17. Pivovarova NB, Andrews SB. Calcium-dependent mitochondrial function and dysfunction in neurons. Febs J 2010;277:3622-3636.

18. Starkov AA, Chinopoulos C, Fiskum G. Mitochondrial calcium and oxidative stress as mediators of ischemic brain injury. Cell Calcium 2004;36:257-264.

19. Kinnally KW, Peixoto PM, Ryu SY, Dejean LM. Is mPTP the gatekeeper for necrosis, apoptosis, or both? Biochim Biophys Acta 2010. doi:10.1016/j.bbamcr.2010.09.13.

20. Hirsch T, Susin SA, Marzo I, Marchetti P, Zamzami N, Kroemer G. Mitochondrial permeability transition in apoptosis and necrosis [In Process Citation]. Cell Biol Toxicol 1998;14:141-145.

21. Lemasters JJ, Nieminen AL, Qian T, et al. The mitochondrial permeability transition in cell death: a common mechanism in necrosis, apoptosis and autophagy. Biochim Biophys Acta 1998;1366:177-196.

22. Lemasters JJ, Qian T, Elmore SP, et al. Confocal microscopy of the mitochondrial permeability transition in necrotic cell killing, apoptosis and autophagy. Biofactors 1998;8:283-285.

23. Vandenabeele P, Galluzzi L, Vanden Berghe T, Kroemer G. Molecular mechanisms of necroptosis: an ordered cellular explosion. Nat Rev Mol Cell Biol 2010;11:700-714.

24. Crompton M. On the involvement of mitochondrial intermembrane junctional complexes in apoptosis. Curr Med Chem 2003;10:1473-1484.

25. Crompton M, Barksby E, Johnson N, Capano M. Mitochondrial intermembrane junctional complexes and their involvement in cell death. Biochimie 2002;84:143-152.

26. Halestrap AP, McStay GP, Clarke SJ. The permeability transition pore complex: another view. Biochimie 2002;84:153-166.

27. Zorov DB, Juhaszova M, Yaniv Y, Nuss HB, Wang S, Sollott SJ. Regulation and pharmacology of the mitochondrial permeability transition pore. Cardiovasc Res 2009;83:213-225.

28. Nicholls DG, Ward MW. Mitochondrial membrane potential and neuronal glutamate excitotoxicity: mortality and millivolts. Trends Neurosci 2000;23:166-174.

29. Szabo I, Bernardi P, Zoratti M. Modulation of the mitochondrial megachannel by divalent cations and protons. J Biol Chem 1992;267:2940-2946.

30. Szabo I, Zoratti M. The giant channel of the inner mitochondrial membrane is inhibited by cyclosporin A. J Biol Chem 1991;266:33763379.

31. Szabo I, Zoratti M. The mitochondrial megachannel is the permeability transition pore. J Bioenerg Biomembr 1992;24:111-117.

32. Zoratti M, Szabo I. The mitochondrial permeability transition. Biochim Biophys Acta 1995;1241:139-176.

33. Sesso A, Marques MM, Monteiro MM, et al. Morphology of mitochondrial permeability transition: morphometric volumetry in apoptotic cells. Anat Rec A Discov Mol Cell Evol Biol 2004;281:1337-1351.

34. Christofferson DE, Yuan J. Necroptosis as an alternative form of programmed cell death. Curr Opin Cell Biol 2010;22:263-268.

35. Duprez L, Wirawan E, Vanden Berghe T, Vandenabeele P. Major cell death pathways at a glance. Microbes Infect 2009;11:1050-1062. 
36. Green DR, Evan GI. A matter of life and death. Cancer Cell 2002;1:19-30.

37. McEwen ML, Springer JE. The biology of capsases in central nervous system trauma. In: Banik N,Lajtha A, eds. Handbook of Neurochemistry and Molecular Neurobiology: Neural Protein Metabolism and Function, 3 rd ed. New York: Springer, 2007:515-550.

38. Wang X. The expanding role of mitochondria in apoptosis. Genes Dev 2001;15:2922-2933.

39. Eguchi Y, Shimizu S, Tsujimoto Y. Intracellular ATP levels determine cell death fate by apoptosis or necrosis. Cancer Res 1997; 57:1835-1840.

40. Formigli L, Papucci L, Tani A, et al. Aponecrosis: morphological and biochemical exploration of a syncretic process of cell death sharing apoptosis and necrosis. J Cell Physiol 2000;182:41-49.

41. Leist M, Single B, Castoldi AF, Kuhnle S, Nicotera P. Intracellular adenosine triphosphate (ATP) concentration: a switch in the decision between apoptosis and necrosis. J Exp Med 1997;185:1481-1486.

42. Nicotera P, Leist M, Ferrando-May E. Intracellular ATP, a switch in the decision between apoptosis and necrosis. Toxicol Lett 1998;102-103:139-142.

43. Jin $\mathrm{Y}, \mathrm{McEwen}$ ML, Nottingham SA, et al. The mitochondrial uncoupling agent 2,4-dinitrophenol improves mitochondrial function, attenuates oxidative damage, and increases white matter sparing in the contused spinal cord. J Neurotrauma 2004;21:13961404.

44. McEwen ML, Sullivan PG, Springer JE. Pretreatment with the cyclopsorin derivative, NIM811, improves the function of synaptic mitochondria following spinal cord contusion in rats. J Neurotrauma 2007;24:613-624.

45. Patel SP, Sullivan PG, Lyttle TS, Rabchevsky AG. Acetyl-Lcarnitine ameliorates mitochondrial dysfunction following contusion spinal cord injury. J Neurochem 2010;114:291-301.

46. Patel SP, Sullivan PG, Pandya JD, Rabchevsky AG. Differential effects of the mitochondrial uncoupling agent, 2,4-dinitrophenol, or the nitroxide antioxidant, Tempol, on synaptic or nonsynaptic mitochondria after spinal cord injury. J Neurosci Res 2009;87:130-140.

47. Sullivan PG, Krishnamurthy S, Patel SP, Pandya JD, Rabchevsky AG. Temporal characterization of mitochondrial bioenergetics after spinal cord injury. J Neurotrauma 2007;24:991-999.

48. Maragos WF, Korde AS. Mitochondrial uncoupling as a potential therapeutic target in acute central nervous system injury. J Neurochem 2004;91:257-262.

49. Sullivan PG, Springer JE, Hall ED, Scheff SW. Mitochondrial uncoupling as a therapeutic target following neuronal injury. J Bioenerg Biomembr 2004;36:353-356.

50. Xiong Y, Singh IN, Hall ED. Tempol protection of spinal cord mitochondria from peroxynitrite-induced oxidative damage. Free Radic Res 2009;43:604-612.

51. Calabrese V, Ravagna A, Colombrita C, et al. Acetylcarnitine induces heme oxygenase in rat astrocytes and protects against oxidative stress: involvement of the transcription factor Nrf2. J Neurosci Res 2005;79:509-521.

52. Clark JB, Nicklas WJ. The metabolism of rat brain mitochondria. Preparation and characterization. J Biol Chem 1970;245:47244731

53. Nicklas WJ, Clark JB, Williamson JR. Metabolism of rat brain mitochondria. Studies on the potassium ion-stimulated oxidation of pyruvate. Biochem J 1971;123:83-95.

54. Martin E, Rosenthal RE, Fiskum G. Pyruvate dehydrogenase complex: metabolic link to ischemic brain injury and target of oxidative stress. J Neurosci Res 2005;79:240-247.

55. Paradies G, Petrosillo G, Gadaleta MN, Ruggiero FM. The effect of aging and acetyl-L-carnitine on the pyruvate transport and oxidation in rat heart mitochondria. FEBS Lett 1999;454:207-209.

56. Petruzzella V, Baggetto LG, Penin F, et al. In vivo effect of acetylL-carnitine on succinate oxidation, adenine nucleotide pool and lipid composition of synaptic and non-synaptic mitochondria from cerebral hemispheres of senescent rats. Arch Gerontol Geriatr 1992;14:131-144.
57. Anonymous. Acetyl-L-carnitine. Altern Med Rev 1999;4:438-441.

58. Lewen A, Matz P, Chan PH. Free radical pathways in CNS injury. J Neurotrauma 2000;17:871-890.

59. Fiskum G. Mitochondrial participation in ischemic and traumatic neural cell death. J Neurotrauma 2000;17:843-855.

60. Starkov A, Fiskum G, Chinopoulos C, et al. Mitochondrial alphaketoglutarate dehydrogenase complex generates reactive oxygen species. J Neurosci 2004;24:7779-7788.

61. Anderson DK, Waters TR, Means ED. Pretreatment with alpha tocopherol enhances neurologic recovery after experimental spinal cord compression injury. J Neurotrauma 1988;5:61-67.

62. Al Jadid MS, Robert A, Al-Mubarak S. The efficacy of alphatocopherol in functional recovery of spinal cord injured rats: an experimental study. Spinal Cord 2009;47:662-667.

63. Bozbuga M, Izgi N, Canbolat A. The effects of chronic alphatocopherol administration on lipid peroxidation in an experimental model of acute spinal cord injury. Neurosurg Rev 1998;21:36-42.

64. Sheu SS, Nauduri D, Anders MW. Targeting antioxidants to mitochondria: a new therapeutic direction. Biochim Biophys Acta 2006;1762:256-265.

65. Miller ER, 3 rd, Pastor-Barriuso R, Dalal D, Riemersma RA, Appel LJ, Guallar E. Meta-analysis: high-dosage vitamin E supplementation may increase all-cause mortality. Ann Intern Med 2005;142:37-46.

66. Kohno M. Applications of electron spin resonance spectrometry for reactive oxygen species and reactive nitrogen species research. J Clin Biochem Nutr 2010;47:1-11.

67. Kopani M, Celec P, Danisovic L, Michalka P, Biro C. Oxidative stress and electron spin resonance. Clin Chim Acta 2006;364:61-66.

68. Farooque M, Olsson Y, Hillered L. Pretreatment with alphaphenyl-N-tert-butyl-nitrone (PBN) improves energy metabolism after spinal cord injury in rats. J Neurotrauma 1997;14:469-476.

69. Li GL, Farooque M, Holtz A, Olsson Y. Effects of alpha-phenylN-tert-butyl nitrone (PBN) on compression injury of rat spinal cord. Free Radic Res 1997;27:187-196.

70. Hillard VH, Peng H, Zhang Y, et al. Tempol, a nitroxide antioxidant, improves blocomotor and histological outcomes after spinal cord contusion in rats. J Neurotrauma 2004;21:1405-1414.

71. Xiong Y, Hall ED. Pharmacological evidence for a role of peroxynitrite in the pathophysiology of spinal cord injury. Exp Neurol 2009;216:105-114.

72. Grilli M, Pizzi M, Memo M, Spano P. Neuroprotection by aspirin and sodium salicylate through blockade of NF-kappaB activation. Science 1996;274:1383-1385.

73. Ko HW, Park KY, Kim H, et al. Ca2+-mediated activation of cJun N-terminal kinase and nuclear factor kappa B by NMDA in cortical cell cultures. J Neurochem 1998;71:1390-1395.

74. Noh J, Lee ES, Chung JM. The novel NMDA receptor antagonist, 2-hydroxy-5-(2,3,5,6-tetrafluoro-4-trifluoromethyl-benzylamino)benzoic acid, is a gating modifier in cultured mouse cortical neurons. J Neurochem 2009;109:1261-1271.

75. Gionchetti P, Guarnieri C, Campieri M, et al. Scavenger effect of sulphasalazine (SASP), 5-aminosalicylic acid (5-ASA), and olsalazine (OAZ). Gut 1990;31:730-731.

76. Ryu BR, Lee YA, Won SJ, et al. The novel neuroprotective action of sulfasalazine through blockade of NMDA receptors. J Pharmacol Exp Ther 2003;305:48-56.

77. Springer JE, Rao RR, Lim HR, et al. The functional and neuroprotective actions of Neu2000, a dual-acting pharmacological agent, in the treatment of acute spinal cord injury. J Neurotrauma 2010;27:139-149.

78. Sullivan PG, Rabchevsky AG, Keller JN, et al. Intrinsic differences in brain and spinal cord mitochondria: Implication for therapeutic interventions. J Comp Neurol 2004;474:524-534.

79. Kelso GF, Porteous CM, Hughes G, et al. Prevention of mitochondrial oxidative damage using targeted antioxidants. Ann N Y Acad Sci 2002;959:263-274.

80. Kelso GF, Porteous CM, Coulter CV, et al. Selective targeting of a redox-active ubiquinone to mitochondria within cells: antioxidant and antiapoptotic properties. J Biol Chem 2001;276:4588-4596.

81. Dhanasekaran A, Kotamraju S, Karunakaran C, et al. Mitochondria superoxide dismutase mimetic inhibits peroxide-induced 
oxidative damage and apoptosis: role of mitochondrial superoxide. Free Radic Biol Med 2005;39:567-583.

82. Cassina P, Cassina A, Pehar M, et al. Mitochondrial dysfunction in SOD1G93A-bearing astrocytes promotes motor neuron degeneration: prevention by mitochondrial-targeted antioxidants. J Neurosci 2008;28:4115-4122.

83. Basso E, Fante L, Fowlkes J, Petronilli V, Forte MA, Bernardi P. Properties of the permeability transition pore in mitochondria devoid of Cyclophilin D. J Biol Chem 2005;280:18558-18561.

84. Nakagawa T, Shimizu S, Watanabe T, et al. Cyclophilin Ddependent mitochondrial permeability transition regulates some necrotic but not apoptotic cell death. Nature 2005;434:652658.

85. Schinzel AC, Takeuchi O, Huang Z, et al. Cyclophilin D is a component of mitochondrial permeability transition and mediates neuronal cell death after focal cerebral ischemia. Proc Natl Acad Sci U S A 2005;102:12005-12010.

86. Baines CP, Kaiser RA, Purcell NH, et al. Loss of cyclophilin D reveals a critical role for mitochondrial permeability transition in cell death. Nature 2005;434:658-662.

87. Brustovetsky N, Brustovetsky T, Jemmerson R, Dubinsky JM. Calcium-induced cytochrome $\mathrm{c}$ release from CNS mitochondria is associated with the permeability transition and rupture of the outer membrane. J Neurochem 2002;80:207-218.

88. Chai J, Du C, Wu JW, Kyin S, Wang X, Shi Y. Structural and biochemical basis of apoptotic activation by Smac/DIABLO. Nature 2000;406:855-862.

89. Du C, Fang M, Li Y, Li L, Wang X. Smac, a mitochondrial protein that promotes cytochrome c-dependent caspase activation by eliminating IAP inhibition. Cell 2000;102:33-42.

90. Li LY, Luo X, Wang X. Endonuclease G is an apoptotic DNase when released from mitochondria. Nature 2001;412:95-99.

91. Petronilli V, Penzo D, Scorrano L, Bernardi P, Di Lisa F. The mitochondrial permeability transition, release of cytochrome $\mathrm{c}$ and cell death. Correlation with the duration of pore openings in situ. $\mathrm{J}$ Biol Chem 2001;276:12030-12034.

92. van Loo G, Schotte P, van Gurp M, et al. Endonuclease G: a mitochondrial protein released in apoptosis and involved in caspase-independent DNA degradation. Cell Death Differ 2001;8:1136-1142.

93. Cande C, Cohen I, Daugas E, et al. Apoptosis-inducing factor (AIF): a novel caspase-independent death effector released from mitochondria. Biochimie 2002;84:215-222.

94. Argaud L, Gateau-Roesch O, Muntean D, et al. Specific inhibition of the mitochondrial permeability transition prevents lethal reperfusion injury. J Mol Cell Cardiol 2005;38:367-374.

95. Friberg H, Ferrand-Drake M, Bengtsson F, Halestrap AP, Wieloch T. Cyclosporin A, but not FK 506, protects mitochondria and neurons against hypoglycemic damage and implicates the mitochondrial permeability transition in cell death. J Neurosci 1998; 18:5151-5159.

96. Gomez L, Thibault H, Gharib A, et al. Inhibition of mitochondrial permeability transition improves functional recovery and reduces mortality following acute myocardial infarction in mice. Am J Physiol Heart Circ Physiol 2007;293:H1654-1661.

97. Li PA, Kristian T, He QP, Siesjo BK. Cyclosporin A enhances survival, ameliorates brain damage, and prevents secondary mitochondrial dysfunction after a 30-minute period of transient cerebral ischemia. Exp Neurol 2000;165:153-163.

98. Matsumoto S, Friberg H, Ferrand-Drake M, Wieloch T. Blockade of the mitochondrial permeability transition pore diminishes infarct size in the rat after transient middle cerebral artery occlusion. J Cereb Blood Flow Metab 1999;19:736-741.

99. Piot C, Croisille P, Staat P, et al. Effect of cyclosporine on reperfusion injury in acute myocardial infarction. $\mathrm{N}$ Engl J Med 2008;359:473-481.

100. Scheff SW, Sullivan PG. Cyclosporin A significantly ameliorates cortical damage following experimental traumatic brain injury in rodents. J Neurotrauma 1999;16:783-792.

101. Sullivan PG, Rabchevsky AG, Hicks RR, Gibson TR, FletcherTurner A, Scheff SW. Dose-response curve and optimal dosing regimen of cyclosporin A after traumatic brain injury in rats. Neuroscience 2000;101:289-295.
102. Sullivan PG, Thompson MB, Scheff SW. Cyclosporin A attenuates acute mitochondrial dysfunction following traumatic brain injury. Exp Neurol 1999;160:226-234.

103. Uchino H, Minamikawa-Tachino R, Kristian T, et al. Differential neuroprotection by cyclosporin A and FK506 following ischemia corresponds with differing abilities to inhibit calcineurin and the mitochondrial permeability transition. Neurobiol Dis 2002;10:219233.

104. Empey PE, McNamara PJ, Young B, Rosbolt MB, Hatton J. Cyclosporin A disposition following acute traumatic brain injury. J Neurotrauma 2006;23:109-116.

105. Diaz-Ruiz A, Rios C, Duarte I, et al. Cyclosporin-A inhibits lipid peroxidation after spinal cord injury in rats. Neurosci Lett 1999;266:61-64.

106. Diaz-Ruiz A, Rios C, Duarte I, et al. Lipid peroxidation inhibition in spinal cord injury: cyclosporin-A vs methylprednisolone. Neuroreport 2000;11:1765-1767.

107. Ibarra A, Correa D, Willms K, et al. Effects of cyclosporin-A on immune response, tissue protection and motor function of rats subjected to spinal cord injury. Brain Res 2003;979:165-178.

108. Ibarra A, Guizar-Sahagun G, Correa D, et al. Alteration of cyclosporin-A pharmacokinetics after experimental spinal cord injury. J Neurotrauma 1996;13:267-272.

109. Ibarra A, Reyes J, Martinez S, et al. Use of cyclosporin-A in experimental spinal cord injury: design of a dosing strategy to maintain therapeutic levels. J Neurotrauma 1996;13:569-572.

110. Rabchevsky AG, Fugaccia I, Sullivan PG, Scheff SW. Cyclosporin A treatment following spinal cord injury to the rat: behavioral effects and stereological assessment of tissue sparing. J Neurotrauma 2001;18:513-522.

111. Sullivan PG, Rabchevsky AG, Waldmeier PC, Springer JE. Mitochondrial permeability transition in CNS trauma: cause or effect of neuronal cell death? J Neurosci Res 2005;79:231-239.

112. Hansson MJ, Mattiasson G, Mansson R, et al. The nonimmunosuppressive cyclosporin analogs NIM811 and UNIL025 display nanomolar potencies on permeability transition in brain-derived mitochondria. J Bioenerg Biomembr 2004;36:407-413.

113. Waldmeier PC, Feldtrauer JJ, Qian T, Lemasters JJ. Inhibition of the mitochondrial permeability transition by the nonimmunosuppressive cyclosporin derivative NIM811. Mol Pharmacol 2002;62:22-29.

114. Waldmeier PC, Zimmermann K, Qian T, Tintelnot-Blomley M, Lemasters JJ. Cyclophilin D as a drug target. Curr Med Chem 2003;10:1485-1506.

115. Chatterji U, Bobardt M, Selvarajah S, et al. The isomerase active site of cyclophilin A is critical for hepatitis $\mathrm{C}$ virus replication. J Biol Chem 2009;284:16998-17005.

116. Goto K, Watashi K, Murata T, Hishiki T, Hijikata M, Shimotohno $\mathrm{K}$. Evaluation of the anti-hepatitis $\mathrm{C}$ virus effects of cyclophilin inhibitors, cyclosporin A, and NIM811. Biochem Biophys Res Commun 2006;343:879-884.

117. Ma S, Boerner JE, TiongYip C, et al. NIM811, a cyclophilin inhibitor, exhibits potent in vitro activity against hepatitis $\mathrm{C}$ virus alone or in combination with alpha interferon. Antimicrob Agents Chemother 2006;50:2976-2982.

118. Coelmont L, Kaptein S, Paeshuyse J, et al. Debio 025, a cyclophilin binding molecule, is highly efficient in clearing hepatitis $\mathrm{C}$ virus (HCV) replicon-containing cells when used alone or in combination with specifically targeted antiviral therapy for HCV (STAT-C) inhibitors. Antimicrob Agents Chemother 2009;53:967-976.

119. Flisiak R, Feinman SV, Jablkowski M, et al. The cyclophilin inhibitor Debio 025 combined with PEG IFNalpha2a significantly reduces viral load in treatment-naive hepatitis $\mathrm{C}$ patients. Hepatology 2009;49:1460-1468.

120. Mathy JE, Ma S, Compton T, Lin K. Combinations of cyclophilin inhibitor NIM811 with hepatitis C Virus NS3-4A Protease or NS5B polymerase inhibitors enhance antiviral activity and suppress the emergence of resistance. Antimicrob Agents Chemother 2008;52:3267-3275.

121. Raisky O, Gomez L, Chalabreysse L, et al. Mitochondrial permeability transition in cardiomyocyte apoptosis during acute graft rejection. Am J Transplant 2004;4:1071-1078. 
122. Fox DA, Poblenz AT, He L, Harris JB, Medrano CJ. Pharmacological strategies to block rod photoreceptor apoptosis caused by calcium overload: a mechanistic target-site approach to neuroprotection. Eur J Ophthalmol 2003;13(suppl 3:S44-S456.

123. Popovich PG, Horner PJ, Mullin BB, Stokes BT. A quantitative spatial analysis of the blood-spinal cord barrier. I. Permeability changes after experimental spinal contusion injury. Exp Neurol 1996;142:258-275.

124. Noble LJ, Wrathall JR. Distribution and time course of protein extravasation in the rat spinal cord after contusive injury. Brain Res 1989;482:57-66.

125. Ravikumar R, McEwen ML, Springer JE. Post-treatment with the cyclosporin derivative, NIM811, reduced indices of cell death and increased the volume of spared tissue in the acute period following spinal cord contusion. J Neurotrauma 2007;24:16181630 .
126. Korde AS, Pettigrew LC, Craddock SD, Pocernich CB, Waldmeier PC, Maragos WF. Protective effects of NIM811 in transient focal cerebral ischemia suggest involvement of the mitochondrial permeability transition. J Neurotrauma 2007;24:895-908.

127. Hokari M, Kuroda S, Iwasaki Y. Pretreatment with the ciclosporin derivative NIM811 reduces delayed neuronal death in the hippocampus after transient forebrain ischaemia. J Pharm Pharmacol 2010;62:485-490.

128. Mbye LH, Singh IN, Sullivan PG, Springer JE, Hall ED. Attenuation of acute mitochondrial dysfunction after traumatic brain injury in mice by NIM811, a non-immunosuppressive cyclosporin A analog. Exp Neurol 2008;209:243-253.

129. Mbye LH, Singh IN, Carrico KM, Saatman KE, Hall ED. Comparative neuroprotective effects of cyclosporin $\mathrm{A}$ and NIM811, a nonimmunosuppressive cyclosporin A analog, following traumatic brain injury. J Cereb Blood Flow Metab 2009;29:87-97. 